method used - the types of cell and how robust they are, what features need to be followed, even the size of the image files that will be collected (see 'A long-term live-cell commitment').

The most important decision is usually what type of light to use. Fluorescent tags can be linked to the expression of particular proteins to indicate specific biological activity or the production of an oncogene, for example. But too much fluorescence-activating light can trigger damage that prevents cells from growing - a phenomenon known as phototoxicity.

Even with cells that resist phototoxicity, there are image-processing considerations. If viewed too often, fluorescent proteins can fade and become invisible. Overcoming such photobleaching requires the subtraction of background fluorescence and correcting for the fact that because the sample is not perfectly flat, levels of illumination vary across the field of view.

Unlike fluorescence microscopy, phase-contrast imaging produces black and white pictures using less damaging wavelengths of light. But the technique reveals only the general cell shape, rather than the presence of a particular protein. Researchers often combine the two methods by, for example, taking a fluorescent image every hour and a phase-contrast image every ten minutes, but even that compromise must be planned carefully. Some instruments that are great for fluorescence microscopy perform less effectively at bright-field microscopy, explains Schlaeger.

\section{Keeping watch}

Keeping cells alive requires a much more delicate balancing act than finding the right mix or amount of photons. Even if cells aren't perturbed by the imaging set-up, they still need fresh culture media and the removal of waste
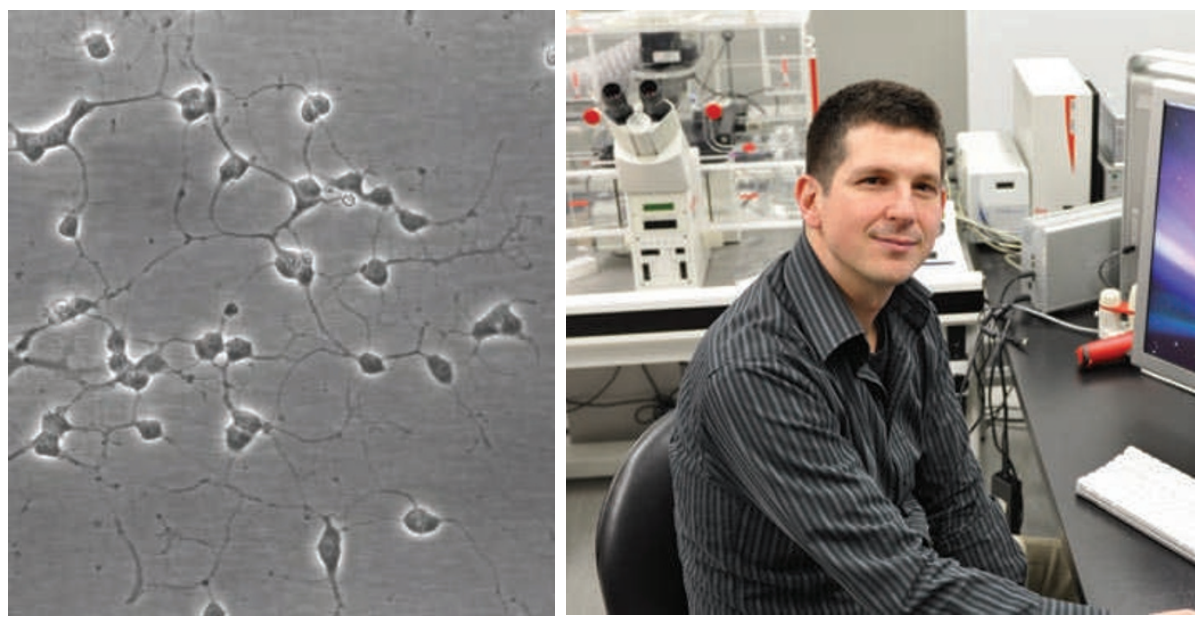

Michel Cayouette (right) uses long-term imaging to predict which retinal progenitors will produce neurons.

products. Plus, any continuous imaging study that lasts for more than an hour will probably require a system with built-in environmental controls for temperature, humidity and gas concentration - and maintaining the right conditions for cells often requires special care for microscopes.

For example, to avoid creating air currents that could blur an image when cells are kept at standard $37^{\circ} \mathrm{C}$, microscopes for studying live cells have to maintain the lens at the same temperature as the culture. Environmental chambers are available for all high-end microscopes, either from the microscope manufacturer or from third parties, but none works as well as an incubator, say researchers. Temple and her graduate students nicknamed an early model 'the Sahara' because it caused cells to dry out so quickly.

The commercially available products have improved, says Jin-Wu Tsai, who studies cultured brain slices in Arnold Kriegstein's lab at the University of California, San Francisco.
"A few years ago, we built our own incubator on top of the microscope. Now you have lots of options," he says. With these newer commercial tools, "we can just keep the culture dish on the stage of the microscope, and the software allows us to take images every ten or fifteen minutes".

Although he doesn't have to transfer samples, Tsai is still tethered to his microscope, keeping watch on its confocal image. The brain slices he studies flatten over time, making the image go out of focus, and it doesn't matter how healthy the cells are if the data collected from them aren't usable. Prototype microscopes with autofocus have been introduced. They might be reliable for flat culture, Tsai says, but he doesn't trust them to work in thick brain slices yet.

In addition to keeping tabs on the brain slices themselves, Tsai has to monitor every facet of the experiment. If the conditions are just slightly off — say, a shift in $\mathrm{pH}$, or a slight increase in carbon dioxide - the neurons stop

\title{
A SOFTWARE SPOT
}

Computerized robotics are already easing lab-based wet tasks such as feeding cells and changing media. Several vendors now sell programs that can track cells in flat culture, keeping them in focus and in the field of view.

The latest version of the Nikon BioStation CT can 'memorize' the positions of non-motile cells before a plate is removed for media exchange, and can then continue tracking them when the plate is replaced, avoiding the 'image jiggle' that would disrupt statistical analysis, says Ned Jastromb, a senior application manager at Nikon Instruments in Melville, New York. It also integrates a calendar function with a robotic system that slides culture plates in and out of an imaging area on schedule, allowing one instrument to run several long-term experiments.

But software is poised to solve a wider range of problems. By combining a fast image-acquisition program with a noise-reducing algorithm that compares consecutive images, John Sedat at the University of California, San Francisco, and his colleagues decreased the amount of light needed to image yeast cell division by several orders of magnitude ${ }^{5}$. Advances in fully automated cell identification and tracking, and modern continuous cell-imaging techniques can outperform traditional manual methods ${ }^{6}$. Historically, software advances have spread slowly because programs designed to follow a particular cell type tend not to recognize other types, says Andrew Cohen, a computer engineer at the University of WisconsinMilwaukee.

More broadly, Cohen says he may be on the cusp of solving a problem that plagues many live-cell imaging experiments. Many software programs work only when cells are sparse. That limits the technology because some cells can grow only in dense cultures, and some cells divide many times before producing the desired cell types, in which case a single cell produces hundreds of daughters. By the time the most interesting cells appears, it is impossible to tell which cells they came from. Recently, Cohen found that an algorithm he originally wrote to follow hundreds of organelles within a single cell can be applied to trace neural stem-cell fate. "Our ability to track very highdensity image sequences is going to improve very rapidly," he says.

Larger advances, however, may come less from improvement in technology than from biologists' awareness of what software can do, says Cohen. "Sometimes the biologists start out just wanting to characterize data, and they don't think about the big questions they can ask." 